Int. J. Dev. Biol. 55: 773-780

doi: $10.1387 / \mathrm{ijdb} .113372 \mathrm{je}$

\title{
Connexins: a junctional crossroad to breast cancer
}

\author{
JAMAL A. EL-SAGHIR ${ }^{1}$, ELIA T. EL-HABRE ${ }^{2}$, MARWAN E. EL-SABBAN ${ }^{*, 1}$ and RABIH S. TALHOUK ${ }^{*, 2}$ \\ Departments of ${ }^{1}$ Anatomy, Cell Biology and Physiological Sciences, Faculty of Medicine and \\ ${ }^{2}$ Biology, Faculty of Arts and Sciences, American University of Beirut, Beirut, Lebanon
}

\begin{abstract}
The mammary gland presents a valuable model for developmental studies, spanning the embryonic stage through menarche to menopause. The dynamic remodeling of this gland is orchestrated by cellular heterogeneity, integrating mammogenic, systemic and local cues. Gap junctional intercellular communication provides pivotal cross talk of mammary epithelial cells with the surrounding cells and their local microenvironment. Connexins are involved in regulating normal and pathological mammary gland development, through channel-dependent and channelindependent roles. Modulation of the isoforms of connexins expressed, as well as their differential assembly into connexons and recruitment of a variety of associated partners, contributes to the complexity of signaling relayed at the membrane. This confers context-dependent functions of connexins at different stages of development and carcinogenesis. This review will summarize available knowledge about the functional dynamics of connexins and gap junctions in regulating normal mammary gland development and its pathophysiology.
\end{abstract}

KEY WORDS: mammary gland, connexin, gap junction, differentiation, cancer

\section{Introduction}

Being the organ responsible for milk production in females, the mammary gland is one of the distinctive features characterizing the class "Mammalia". The highly branched architecture of the gland acquired post-puberty and its plasticity that reflects a constant remodeling in response to microenvironmental cues, make it a valuable developmental model to investigate. Rodent and human mammary glands share similar cellular composition of their parenchyma, mainly the epithelial, adipose and other stromal cells (Maller et al., 2010). The most notable difference is in the relative abundance of connective tissue to epithelial cells. The more accessible rodent tissue, with low stromal to epithelial ratio, is thus an attractive surrogate to study breast and cancer development. During mouse embryonic development, mammary epithelial placodes begin to branch from the ectoderm into a rudimentary tree, with relatively restricted growth until puberty. Initial branching of the epithelium thus results in a bilayered ductal structure, whose inner ductal layer harbors the ductal and alveolar luminal cells, and its outer counterpart is mainly composed of myoepithelial cells. At the onset of puberty, secreted systemic hormones induce secondary side branching, where the ductal ends proliferate, expand their lumens by shedding cells and grow into the surrounding adipose tissue, subsiding to minor remodeling during recurrent estrous cycles. Upon pregnancy, full differentiation of the gland induces extensive branching, proliferation and sprouting of the lobulo-alveolar milk-secreting buds at the forefront of the ducts. At this tertiary side branching stage, ductules of mammary epithelial cells take up most of the fat pad. This remodeling supports lactation, when the alveolar luminal cells apically secrete milk into the lumen, where its expulsion is aided by the contractility of an outer layer of myoepithelial cells that surrounds the alveoli. Postweaning, the structure returns to a virgin-like architecture due to the degeneration of the newly acquired structures. This complex process of mammary development is orchestrated by a complex array of microenvironmental cues (Tiede et al., 2011).

Systemic hormones and paracrine factors contribute to the development, homeostasis and functionality of the mammary gland, whose cellular components are spacio-temporally conditioned to express the associated receptors (Brisken et al., 2010). These humoral mammogens cross-talk with local cellular components that express, in a context-dependent manner, extracellular matrix (ECM) components and modifiers such as matrix metalloproteinases (MMPs), a disintegrin and metalloproteinases (ADAMs) and their inhibitors to allow for the gland's flexible remodeling (Khokha et al.,

Abbreviations used in this paper: Cx, connexin; ECM, extracellular matrix; GJ, gap junction; GJIC, gapjunctional intercellular communication; ZO, zonula occludens.

\footnotetext{
*Address correspondence to: Rabih S. Talhouk. Biology, Faculty of Arts and Sciences, American University of Beirut, P.O. Box: 11-0236, Beirut, Lebanon.

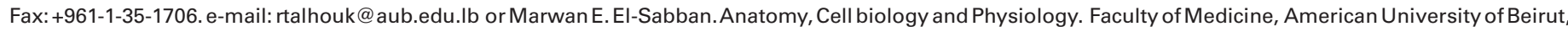
P.O. Box 11-0236, Beirut, Lebanon. Tel: +961-1-35-0000, ext. 4765. Fax: +961-7-44-464. e-mail: me00@aub.edu.lb
} 
2011). More recently, ECM mechanics has also been implicated in the establishment of cellular polarity, proliferation and cell fate determination. The ECM in general and collagen specifically is a mechanosensitive reservoir of growth factors and cytokines that are responsive to proteolytic cleavage that affects the mechanical strength of the milieu, in turn activating/deactivating mechanosensitive mediators such as TGF- $\beta$ (Maller et al., 2010).

On another note, cellular junctions such as hemidesmosomes, tight and adherens junctions exert physical tension contributing to the chemical and physical polarization of the cells. All the tensile forces are choreographed by the cytoskeleton, bidirectionally relaying the cues from and to the transcriptional level. Exposure of mammary epithelial cells to different tensile forces, either imposed during milk suckling, interaction with neighboring cells or more subtle matrix stiffness changes, monitored in 2D versus 3D culture systems, were found to deeply impact cell to cell signaling (Schedin et al., 2011; Mroue et al., 2011).

Tight, adherens, desmosomal and gap junctions are the main epithelial cell-cell junctional complexes, each of which has a distinctive membranous localization and connection to cytoplasmic elements, mediating cellular polarity and function. Nearest to the apex of the epithelial cell are tight junctions, whose dynamic selective permeable barrier to paracellular movement maintains tissue homeostasis, especially in secreting glands. These junctions are composed of transmembrane occludin and claudins associating with cytoplasmic partner proteins such as the Zona Occludens (ZO) family members, and initiate numerous signaling cascades to the cytoskeleton and the nucleus (Brennan et al., 2010). These modulating partners play a role in the differential permeability needed in the lactating mammary gland, regulating adhesion, migration, polarity and differentiation. Apical polarity markers and their association with cytoskeletal elements at cell junctions are critical for the homeostasis of mammary epithelia by affecting the epigenetic makeup and chromatin organization (Lelièvre et al., 2010). Recent work characterized differential expression of claudins during pregnancy, lactation and involution, and alterations of integral membrane proteins, adaptors or signaling partners were also reported in breast cancer context. Responsiveness of the assembly and tightness of these junction barriers to internal and external cues are pivotal in normal cell functioning and in coordinating synchronous milk secretion (Lanigan et al., 2007).

Adherens junctions also reflect the same complexity and dual function, by not only mediating intercellular adhesion but also by scaffolding the assembly of polarity markers and initiating downstream signaling. Calcium-dependent cadherins constitute the transmembrane backbone of adherens junctions, which are divided into classical and desmosomal cadherins, associating respectively with actin and intermediate filaments. Homophilic assembly of apposing cadherins at the membranes of adjacent cells mediates the recruitment of cytoplasmic signaling partners and contributes to the maintenance of mammary tissue integrity during development and lactation. In the mammary ducts, E-cadherin is expressed in the luminal epithelial cells, whereas P-cadherin is found in the myoepithelial layer. Blocking E-cadherin inhibits growth of the epithelial tissue by interfering with downstream signaling pathways initiated at the junctional complex (Lanigan et al., 2007).

Cellular junctions are of paramount importance to the differentiation status of mammary epithelial cells and gap junctions are no exception. In fact, gap junctions' contribution to mammary differentiation and pathophysiology gained recognition not only by serving as a nexus joining the cytosols of adjacent cells, but also by complexing with adaptor proteins and polarity markers and relaying cues into downstream signaling cascades.

Gap junctions are clusters of apposing hemichannels joining two adjacent cells. Each hemichannel is an oligomer of six transmembrane connexin proteins, with divergent cytoplasmic C-terminus domains mediating differential binding affinities and functions among the different members. Twenty different connexin genes were reported in mice and 21 in humans. Connexin nomenclature features the molecular weight of the isoform $(\mathrm{kDa})$ to convey the type, i.e., Cx32 denotes the connexin with the molecular weight $32 \mathrm{kDa}$. Similar and different connexin isoforms can join forming homomeric and heteromeric hemichannels, respectively. Similar hemichannels can couple with those present on adjacent cells, via homotypic or otherwise heterotypic assembly, forming channels, whose aggregation defines gap junctions. Since many connexin isoforms can be expressed at once in the same cell, these heterogeneous junctions allow differential conductance of intercellular exchange of molecules less than $1.5 \mathrm{kDa}$ in molecular weight. With recent revelation of complex translational and posttranslational mechanisms regulating connexin function and the growing myriad of its interacting proteins including cytoskeletal elements, junctional proteins and enzymes, gap junctions are now perceived not only as channels between neighboring cells, but as signaling complexes that regulate cell function and transformation. Several elements regulating and mediating the signaling downstream of adherens and tight junctions are also associated with gap junctional plaques, underlining the signaling potential of the latter and the cross-regulation of different junctions. For instance, connexin associations with cytoskeletal elements are not only pivotal for their proper transport and localization but also for allowing communication with other junctions. Several polarity markers and enzymes shuttle between the different complexes and contribute to the common pool of "junction-associated molecules" stressing global signal integration yet junction-dependent functions that contribute to mammary epithelial cell differentiation (Dbouk et al., 2009; Mroue et al., 2011).

\section{Gap junctions in normal mammary tissue}

Given the dynamic nature of the mammary gland, investigation of stage-specific gap junctional regulation, ranging from connexin expression to the recruitment of specific associated partners, is crucial for our understanding of normal gland development, tissuespecific function and transformation.

\section{Spatial and temporal expression of connexins}

Among the connexin family, Cx26 and Cx43 were identified as the only connexins expressed in human mammary epithelial cells. Normal breast samples, obtained from reduction mammoplasties, showed that Cx26 was expressed between the luminal cells whereas Cx43 was localized to the myoepithelial cells of the ducts. Although their expression and localization was cell type-specific, both connexins were subject to regulation. Cx26 expression was cell cycle-dependent, suggesting a role for Cx26 in luminal cell proliferation, while Cx43 expression was more constant, suggesting a role for $\mathrm{Cx} 43$ in myoepithelial cell differentiation (McLachlan et al., 2007). The temporal pattern of connexin expression in the 
human mammary gland is not well-characterized due to the difficulty in obtaining normal human breast tissue samples at different developmental stages. As an alternative, the spatial and temporal expression of connexins and their role in rodent mammary gland development was extensively studied, given that connexin genes are highly conserved in both species with respect to their high degree of sequence identity (El-Sabban et al., 2003a; McLachlan et al., 2007; Söhl and Willecke 2004). In addition to Cx26 and Cx43 characterized in the human mammary gland, Cx32 and Cx30 were reported in the mouse mammary gland (Talhouk et al., 2005).

Many studies have reported the modulation of connexin expression during pregnancy, lactation and involution in the rodent mammary gland. In fact, Talhouk et al., (2005) showed that Cx26 and $\mathrm{C} \times 32$, expressed at the basolateral borders of luminal cells, were detected at all developmental stages of the gland. Both connexins had increased expression, at the mRNA and protein levels, during pregnancy; they peaked at lactation and declined at involution to levels comparable to that of the virgin gland. This expression profile of $\mathrm{C} \times 26$ and $\mathrm{C} \times 32$ peaking in lactation highlights their physiological importance in regulating milk production. Cx32 in particular might play an important role in the mouse mammary gland development since it is rodent-specific and not reported in the human breast (McLachlan et al., 2007). We have shown that Cx30, which is epithelial-cell-specific, was detected at the protein level after day 15 of gestation and peaked at the onset of lactation in the mammary gland of BALB/c mice (Talhouk et al., 2005). Two years later, a report using oligonucleotide microarrays confirmed the abundant expression of $\mathrm{Cx} 30$ during late pregnancy and early lactation. Moreover, the study demonstrated that $\mathrm{Cx} 30$ colocalized with Cx26; however, at parturition, a drop in Cx30 mRNA levels was accompanied by a rise in Cx32 expression, suggesting its involvement at this specific developmental stage of the gland (Locke et al., 2007). Interestingly, another study from our laboratory showed that $\mathrm{Cx} 30$ expression and association at the membrane with $\alpha$-catenin, $\beta$-catenin and ZO-2 proteins was only possible in heterocellular cultures of SCp2 and SCg6, the epithelial and myoepithelial subclones of CID-9 mouse mammary cells, respectively, but not in SCp2 cells dripped with EHS-matrix growth factor-reduced matrigel, suggesting a complex hierarchy for its expression that closely correlates with epithelial differentiation (Talhouk et al., 2008). As for Cx43, while its mRNA expression decreased during mid-pregnancy and lactation, its active phosphorylated form was specifically upregulated during lactation (Talhouk et al., 2005). This is in accordance with previous studies from our laboratory that demonstrated a decrease in Cx43 mRNA expression and a concomitant increase in protein levels in CID-9 mammary cells, grown under differentiating conditions, suggesting post-translational regulation of $\mathrm{Cx} 43$ during mammary gland development and differentiation (El-Sabban et al., 2003b). The localization of Cx43 to myoepithelial cells and its regulation at specific stages of development proposes that it may be vital to myoepithelial function during lactation. However, Talhouk et al., (2005) reported that Cx43 was localized to the myoepithelial-epithelial cell contact regions, suggesting that Cx43 may be expressed by myoepithelial cells and secretory alveolar epithelial cells.

\section{Role of connexins in mammary gland development}

To address the role of connexins at specific stages of mammary gland development, many studies have used knockout and trans- genic mouse models. While Cx26 and Cx43 knockout mice were lethal, mammary gland development was not assessed in Cx30 and Cx32 knockout mice. Using genetically altered mouse strains, a study showed that mice with inactivated $\mathrm{Cx} 32$ and $\mathrm{C} \times 43 \mathrm{KI} 32$ mice, in which $\mathrm{Cx} 43$ was replaced with $\mathrm{Cx} 32$, had normal mammary gland development, whereas loss of Cx26 from mammary epithelium before puberty resulted in abrogated lobuloalveolar development and function during lactation. However, ablation of Cx26 during the latter part of pregnancy in the secretory epithelial cells had no consequences on alveolar development and function, suggesting that $\mathrm{C} \times 32$, and perhaps $\mathrm{C} \times 30$, can compensate for Cx26 loss during later stages of pregnancy and lactation (Bry et al., 2004). In support of this, several studies reported a change in channel composition and permeability at different stages of mammary gland development. At the onset of parturition, heteromeric Cx26-Cx32 hemichannels were formed and shifted predominantly into homomeric $\mathrm{Cx} 32$ channels which had wider pores than the former ones allowing the passage of larger permeants. Taurine, an osmolyte which accumulates in mammary cells during lactation, is required for milk synthesis and was shown to inhibit the molecular permeability of $\mathrm{Cx} 26-\mathrm{Cx} 32$ channels but not that of homomeric $\mathrm{Cx} 32$ nor heteromeric $\mathrm{C} \times 26-\mathrm{C} \times 30 / \mathrm{C} \times 30-\mathrm{C} \times 32$ channels, suggesting an important role for $\mathrm{C} \times 30$ and $\mathrm{C} \times 32$ at this specific developmental stage of the gland (Locke et al., 2000, 2004, 2007). Collectively, these findings show that differential oligomerization of connexins is tightly regulated and plays a major role in mammary gland differentiation.

The mouse model (Gja1 ${ }^{\mathrm{Jr} / \mathrm{H}}$ ), that has an autosomal dominant Cx43 mutation and mimics the human disease of oculodentodigital (ODD), has provided a new insight to the role of Cx43 in mammary gland development and function. In these Cx43 mutant mice, decreased Cx43 protein levels were associated with reduced GJIC in myoepithelial cells. While a delay in mammary gland development was observed, the morphology of the gland did not differ from that of wild type mice at parturition. Interestingly, high levels of milk proteins ( $\beta$-casein and WAP) were detected in Gja1 ${ }^{\mathrm{Jr} /+}$ mice at parturition with a failure to deliver milk to the ducts by oxytocin stimulation, suggesting an essential role of $\mathrm{Cx} 43$ in mammary gland function, particularly in the contraction of myoepithelial cells and ejection of milk (Plante et al., 2008). A recent study by the same group demonstrated that other connexins, adherens and tight junction proteins were unaltered in the $\mathrm{C} \times 43$ mutant mice (Plante et al., 2010a). Our group has established a direct correlation between functional GJIC and mammary epithelial differentiation by studying CID-9 mouse mammary cells and their two sub-clones, SCg6 the myoepithelial-like cells, and SCp2 the epithelial cells that express $\beta$-casein as a marker of functional differentiation in response to lactogenic hormones and in the presence of exogenous basement membrane components. We showed that enhanced GJIC induced partial differentiation of mammary epithelial cells, in the absence of exogenously provided basement membrane (El-Sabban et al., 2003b). It should be noted that Cx-associated proteins that include enzymes, cytoskeletal elements, junctional proteins and others known to regulate $\mathrm{Cx}$ assembly and functions, are highly implicated in the channel-independent role of connexins during mammary gland differentiation (Dbouk et al., 2009). We have previously demonstrated that heterocellular interaction between SCg6 and SCp2 caused an increased association between connexins (Cx32, Cx43 and $\mathrm{Cx30}$ ) on one hand and $\alpha$-catenin and ZO-2 
proteins on the other hand, leading to the recruitment of $\beta$-catenin into the gap junctional complex and preventing its translocation to the nucleus, thereby stabilizing the GJ and contributing to the GJ-induced functional differentiation of mammary epithelial cells and $\beta$-casein expression (Talhouk et al., 2008). Thus, in addition to their role as channels allowing the passage of many important regulatory molecules between adjacent cells, connexins are also implicated in channel-independent functions whereby their context-dependent expression and functional interactions further regulate normal tissue function. Given the role of connexins and their associated-proteins in maintaining normal development and differentiation, it is not surprising that any alteration of their expression or localization may contribute to neoplastic progression in the gland (Mroue et al., 2011).

\section{Gap junctions role in neoplastic mammary gland}

Many studies have classified cancer in the category of connexinrelated pathologies (Laird, 2010). In breast carcinogenesis in particular, addressing the role of connexins in cancer progression has yielded contradictory results; while connexins have long been considered as tumor suppressor genes, recent data indicates that invasive breast carcinomas express high levels of connexins (Naus and Laird, 2010). Since cancer involves multiple steps from the onset to progression and metastasis, the paradoxical roles of connexins in breast carcinogenesis, either as tumor suppressors or enhancers, depend greatly on the stage of carcinogenesis at the primary or metastatic sites.

\section{Role of connexins in breast carcinogenesis}

Since Loewenstein and his colleagues (1966) first made the link between the absence of GJIC and cancer, several studies further demonstrated that breast cancer tissues have altered GJIC which is often associated with aberrant expression or localization of connexins. However, it is generally accepted that impaired GJIC facilitates the release of tumor cells and promotes tumorigenesis at the initial steps of this process, while a functional GJIC is found to be crucial, at later stages, for migrating cells to invade, interact with endothelial cells, intravasate and extravasate (Czyz et al., 2008).

Studies have shown that $\mathrm{Cx} 26$ and $\mathrm{Cx} 43$ were downregulated in human breast cancer cell lines. While some researchers have attributed this reduction in Cx26 expression to hypermethylation of the gene, leading to its inactivation, others have linked it to perturbations in cell-cell adhesion and more specifically, a loss of Ecadherin, or to the activation of oncogenes such as ras and c-erbB-2 in keratinocytes and hepatocytes, respectively. However, the role of these oncogenes in mammary neoplasms connexin expression has not been identified (Locke et al., 1998; McLachlan et al., 2007). In addition, Cx43 expression was found to be reduced in all stages of human breast cancer and carcinogen-induced breast tumors, possibly as a result of cancer-specific stimuli such as the activation of PKC, ErbB2, EGF and src pathways (Carystinos et al., 2001). In a recent report, the role of $\mathrm{Cx} 43$ in breast carcinogenesis was further addressed where Cx43 mutant mice were cross-bred with mice overexpressing ErbB2, an oncogene overexpressed in breast cancer patients, and both spontaneous and carcinogen-induced tumor development were assessed. Although those mice exhibited delayed onset of palpable tumors and developed extensive mammary gland hyperplasia, they had increased metastasis to the lungs
(Plante et al., 2010b). Such findings require re-evaluation of the role of connexins in cancer progression and studying them from a different perspective, as conditional tumor suppressors or even tumor enhancers, depending on the stage of carcinogenesis and the context of their microenvironment. In fact, several studies have reported an increased expression of connexins in breast cancer tissue. Cx26 and Cx43 expression was detected in more than 50\% of the invasive breast carcinomas, as compared to normal tissue samples. Phosphorylation of Cx43 was strongly upregulated in myoepithelial cells and transformed luminal cells of in situ and invasive breast carcinomas, as well as in their associated capillary endothelium. Although connexins were shown to be upregulated in some breast cancers, their localization was cytoplasmic and failed to reach the plasma membrane to fulfill their normal function of GJIC (McLachlan et al., 2007).

At later stages of carcinogenesis, connexins were shown to be highly implicated at intravasation and extravasation sites thereby promoting breast cancer metastatic potential. In fact, increased Cx43 expression was detected in tumor cell/endothelial cell contact areas in vitro and in vivo, where it marked the sites of micrometastases to the lungs. Moreover, a GJIC via Cx43 increased the adhesion of breast cancer cells to lung endothelial cells, whereas such adhesion was reduced in breast cancer cells with a dominant-negative Cx43 mutation, known to exhibit non-functional GJIC, highlighting the importance of Cx43 and GJIC in metastatic homing of breast cancer cells (Elzarrad et al., 2008). Other studies have correlated Cx26 expression to an increased lymphatic vessel invasion, large tumor size and poor prognosis in breast cancer patients (Naoi et al., 2007). Similarly, another group of researchers showed that Cx26 and Cx43 contributed to breast cancer metastasis to lymph nodes where their expression was upregulated when compared to primary tumors, with distinct localization of connexins to the membrane (Kanczuga-Koda et al., 2006). Later on, the same group generated similar results and reported Cx32 increased expression in lymph node metastases. Interestingly, $70 \%$ of Cx26, Cx43 and Cx32-negative primary tumors developed Cx26, Cx43 and Cx32-positive lymph node metastases, suggesting an alteration in connexin protein expression during breast cancer progression resulting in a more malignant phenotype (Kanczuga-Koda et al., 2007). Interestingly, in a recent review by Mroue et al., (2011), the authors emphasized the fact that connexins and GJIC were either reduced or enhanced in different cancer models depending on the stage of tumorigenesis, the type of tissue and the microenvironment of the tumor cell, which led to the notion that connexins might have either tumor suppressive or promoting potentials depending on their cellular and tissue context.

\section{Connexins as breast cancer modulators}

The involvement of connexins in modulating breast carcinogenesis can no longer be strictly looked at from a channel-dependent perspective; in fact, connexins and $\mathrm{Cx}$-associated proteins can mediate different signaling events in a channel-independent context. We will elucidate the gap junction-dependent and independent roles of connexins, as either suppressors or facilitators of breast carcinogenesis.

Gapjunction-dependentroles of connexins in breastcarcinogenesis

Knowing that gap junctions are involved in the intercellular exchange of diverse regulatory molecules that affect normal as 
well as cancer cells, several research groups have reported a gap junction-dependent role of connexins in modulating tumor progression. In fact, two reports showed that the expression of BRMS1, a tumor suppressor gene, in MDA-MB-435 cells resulted in the restoration of GJIC. In the breast cancer cell lines MCF-7 and MDA-MB-435, homotypic GJIC was significantly lower than heterotypic GJIC of either cell line with hFOB, a human osteoblastic cell line; this correlated with increased tumorigenesis and metastatic potential. However, metastasis-suppressed MDA-MB-435/ BRMS1 displayed greater homotypic GJIC than heterotypic GJIC with hFOBs, behaving more like normal breast HTERT cells known to have high degree of homotypic GJIC (Saunders et al., 2001; Kapoor et al., 2004). The GJ-dependent role of Cx26 as a tumor suppressor gene was demonstrated when MCF-7 breast cancer cells, supposedly GJ-deficient, were transfected with Cx26. Their cell-cell communication was restored and their malignant properties characterized by anchorage-independent growth, migration and invasion were reduced (Momiyama et al., 2003).

On the other hand, very few studies have attributed a channeldependent function of connexins in enhancing rather than suppressing breast carcinogenesis. In fact, one report assessed the role of GJIC between breast cancer cells and endothelial cells in breast cancer progression; Connexin 43 expression in GJIC-deficient and non-metastatic HBL100 cells resulted in increased GJIC with endothelial cells and subsequent upregulation of diapedesis through the endothelial monolayer, further supporting the concept of stage-specific role of connexins in breast cancer progression (Pollmann et al., 2005).

\section{Gap junction-independent roles of connexins in breast carcino- genesis}

There is increasing evidence for gap junction-independent roles of connexins in the suppression of breast carcinogenesis. Retroviral delivery of $\mathrm{Cx} 26$ and $\mathrm{Cx} 43$ to breast cancer cell lines that either lack connexin expression (HBL100) or have defect in gap junction assembly (MDA-MB-231) resulted in a dramatic inhibition of tumor growth in vivo independently of the formation of gap junctions. Immunostaining of mammary fat pad xenografts of MDA-MB-231 expressing Cx43 showed that $\mathrm{Cx} 43$ was retained within the cell and not assembled into gap junctional plaques (Qin et al., 2002). The same group investigated the role of Cx26 as a tumor suppressor gene in two independent studies. In the first one, they showed that expression of Cx26 and its GJIC-incompetent variants in MDA-MB-435 cells upregulated anti-angiogenic molecules by both GJIC-dependent and -independent mechanisms (Qin et al., 2003). In their second study, only GJIC-incompetent variants inhibited MDA-MB-435 cell growth, migration and invasion and regulated the expression of several genes involved in adhesion ( $\beta$-integrin) and invasion (MMP-9 and TIMP-1), suggesting a GJIC-independent role of Cx26 in breast cancer suppression (Kalra et al., 2006). Another report showed similar results where MDA-MB-435, stably transfected with human $\mathrm{Cx} 43$, had decreased metastatic potential, which was independent of GJIC, migration or invasion; however, it was related to a decrease in $\mathrm{N}$-cadherin expression and increased sensitivity to apoptosis ( $\mathrm{Li}$ et al., 2008). The tumor suppressive role of connexins was further demonstrated in a three-dimensional culture of MDA-MB-231. Cx26 or Cx43 were shown to decrease the malignant properties of breast cancer cells by promoting mesenchymal to epithelial transition and to regulate the release of pro-angiogenic and anti-angiogenic molecules thereby inhibiting angiogenesis in vitro and in vivo (Shao et al., 2005; McLachlan et al., 2006). Another aspect of channel-independent roles of connexins is mediated by $\mathrm{Cx}$-associated proteins that are known to play essential roles in regulating the assembly and function of gap junctions (Dbouk et al., 2009). This highlights the importance of studying connexins and gap junctions in a context-dependent manner, where the cancer cell type, the tumor microenvironment and the stage of carcinogenesis altogether can regulate connexin expression, localization and interactions, and hence their contribution to cancer progression (Mroue et al., 2011).

\section{Gap junctions and breast cancer drug therapies}

The possibility of connexins involvement at various stages of breast carcinogenesis and the accumulating evidence of most cancers having reduced capacity of GJIC suggests that restoration of GJIC at primary stages of the metastatic cascade might have beneficial effects in breast cancer therapies. However, if one considers connexins to facilitate the advancement of late-stage disease, a reverse strategy must be applied to achieve therapeutic purposes in which connexins are downregulated to inhibit extravasation and metastasis. In fact, gamma linolenic acid (GLA), a polyunsaturated fatty acid known for its anti-proliferative and cytotoxic effects on cancer cells as well as its inhibition of cancer cell motility and invasion, was shown to reduce adhesion of breast (MDA-MB-231) and colon (HT115) cancer cells to endothelial cells. GLA was shown to reverse the effect of HGF/SF, a paracrine factor regulating mitogenesis, motility and morphogenesis, which reduced GJIC and increased cell-cell dissociation/motility in HGF/SF-treated endothelial cells. Therefore, the inhibitory effect of GLA on tumor cell to endothelial cell interaction might be attributed to an improvement of GJIC between endothelial cells (Jiang et al., 1997). Later on, combinational treatment strategies were developed and directed towards multiple mechanisms involved in breast cancer progression. One report studied the effect of Tamoxifen and retinoic acid combination on GJIC in MCF-7 cells, as a possible mediator of their anti-cancer activities. Interestingly, combinational treatment enhanced GJIC with a concomitant membranous localization of Cx26 and Cx43 at cell-cell contacts. At the same time, an inhibition of cancer cell proliferation and a partial reversion of the tumor phenotype, characterized by increased E-cadherin expression, decreased $\mathrm{Bcl}-2$ and c-myc protein levels and reduced telomerase activity, suggest a role of GJIC in mediating the action of Tamoxifen and retinoic acid (Saez et al., 2003). More recently, it was demonstrated that combinational treatment of T47D breast cancer cells with Tamoxifen and PQ1, a substituted quinoline and a gap junction activator, caused a decrease in cancer cell proliferation and colony growth, versus an increase in BAX proapoptotic protein levels, subsequently leading to caspase 3 activation. This suggests that $P Q 1$, by increasing GJIC, allows the intercellular passage and the rapid action of Tamoxifen, thereby potentiating apoptosis of breast cancer cells (Gakhar et al., 2010). The same group showed that a second-generation substituted quinoline, PQ7, has anti-tumor effect on the same cell type by activating GJIC, due to an increase in Cx43 protein expression, thus leading to $50 \%$ reduced colony growth in vitro and $100 \%$ regression of tumor growth in xenograft models (Heiniger et al., 2010). The therapeutic potential of gap junctions in combinational treatments can be partially attributed 
to their "bystander effect" whereby restoration of GJIC between neighboring cells provides a route for drugs to be delivered within a tumor mass and to induce cell death. The "bystander effect" was mostly evaluated in brain tumor gene therapy where the delivery of herpes simplex virus thymidine kinase gene to cancer cells, followed by the administration of the pro-drug ganciclovir, resulted in the formation of a toxic phosphorylated form of the drug. The latter induces cytotoxicity in cancer cells expressing HSV-TK, and in surrounding cells not expressing HSV-TK, only if they are coupled by gap junctions (Mesnil et al., 2000).

\section{Conclusion}

Dynamic cues from the cellular microenvironment including humoral mediators such as growth factors and hormones, cell-cell or cell-ECM interactions are essential for proper function of the mammary gland. Gap junctions, mediating the intercellular transfer of regulatory molecules, are found to be important modulators of normal gland development and differentiation as well as of breast cancer progression. However, connexins, the gapjunction proteins, have important functions beyond gap junctional intercellular communication where they interact with a growing list of associated proteins that generate signals downstream of connexins, adding to the complexity of connexin functions to include a channelindependent aspect. Even though connexins have recently been considered as tumor suppressor genes, they have been shown to play diverse roles in breast carcinogenesis depending on cancer progression stages; whereas at primary tumor sites, connexins are downregulated, they are re-expressed at intravasation and extravasation sites to facilitate the progression of the tumor. In Fig. 1 , we proposed a model for the role of connexins and their associated proteins in mammary differentiation on one hand and in breast cancer progression on the other hand. We have already established a role for the assembly of the GJ complex in inducing functional differentiation of mammary epithelial cells (Talhouk et al., 2008). We speculate that the dissociation of such complex and the loss of GJIC might be involved in the initiation of tumorigenesis, whereas at later stages of the metastatic cascade such as at intravasation/ extravasation sites, this complex has to be reassembled in order to allow breast cancer cells' interaction with endothelial cells, thus facilitating tumor progression. Therefore, a better understanding of the extent of connexin involvement at different stages of breast cancer will be highly beneficial for therapeutic strategies that should aim at upregulating connexins at initial stages of the disease or
A

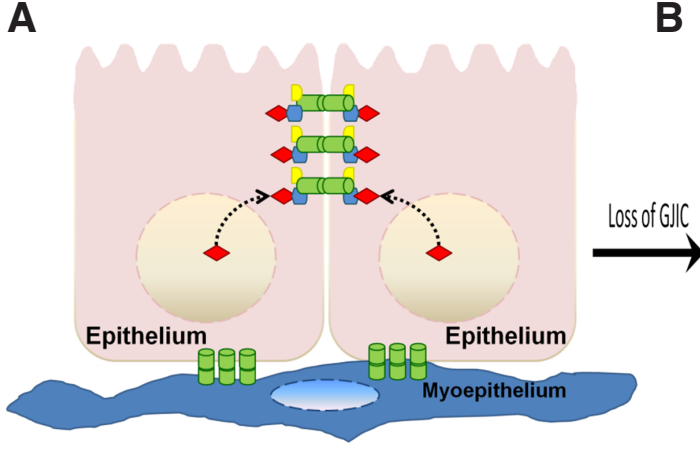

Differentiated cells
B

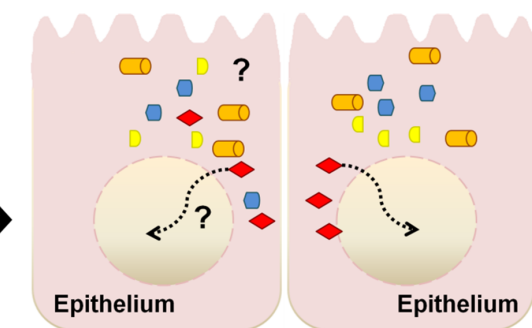

Epithelium

\section{Cancer cells at primary site}

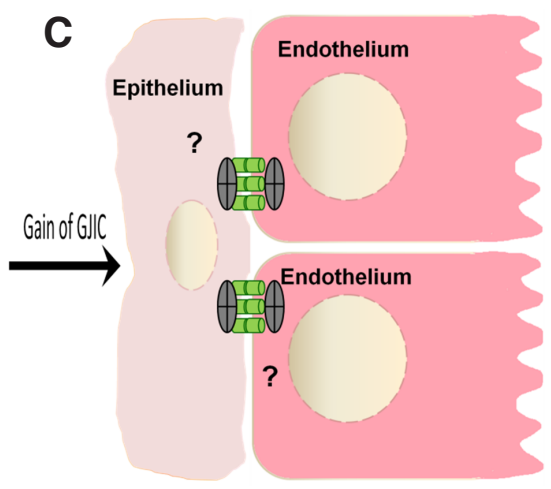

Cancer cell at metastasis

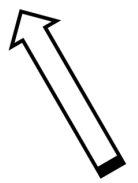

[1] Induce GJIC

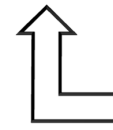

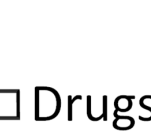

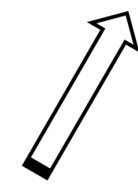

[2] Block GJIC

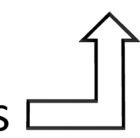

Fig. 1. Proposed dynamics of gap junction (GJ) complex assembly in mammary differentiation and cancer progression. (A) In normal mammary epithelial cells, connexins assemble at the membrane and associate with several signaling molecules including $\alpha$-catenin ( $\square$ ) and ZO-2 (D), thereby recruiting $\beta$-catenin $\bigcirc$ ) from the nucleus to the GJ complex to induce functional differentiation, accompanied with an increased GJIC (Talhouk et al., 2008). (B) At the primary tumor site, connexins are still expressed yet without functional GJIC. The GJ complex dissociates thereby releasing $\beta$ catenin, enabling its recruitment into the nucleus, leading to the loss of communication of tumor cells with surrounding cells and activation of cellular proliferation. (C) As the cancer progresses, invasive epithelial cells regain GJIC with the endothelial barrier, facilitating their intravasation/extravasation. Connexin interactions with other molecules to assemble an invasion conducive protein complex ( $)$ ) could take place to re-stabilize the GJ complex. Recent development in therapeutic approaches target GJIC by [1] inducing the reestablishment the GJ complex to inhibit replication of cancer cells or [2] dissociation of the GJ complex to inhibit cancer cell interaction with endothelial cells at the sites of intravasation/extravasation. 
downregulating them, to inhibit extravasation and subsequent metastasis at advanced stages of the disease. Finally, given the complexity of connexin function and interactions, treatments that target connexins and/or other interacting proteins should be considered in combination with other therapeutic strategies to yield greater benefits for cancer patients.

\section{Acknowledgments}

The authors are grateful for Dr. Samira Kaissi and Dr. Rihab Nasr for critical reading of the manuscript and Ms. Dana Bazzoun for her help in manuscript preparation. This work was supported by the University Research Board and Lebanese National Council for Scientific Research (RST and MES), and Medical Practice Plan (MES).

\section{References}

BRENNAN K, OFFIAH G, MCSHERRY E, and HOPKINS A (2010). Tight junctions: $A$ barrier to the initiation and progression of breast cancer? J Biomed Biotechnol 2010: 460607.

BRISKEN C and O'MALLEY B. (2010). Hormone action in the mammary gland. Cold Spring Harb Perspect Biol 2: a003178.

BRY C, MAASS K, MIYOSHI K, WILLECKE K, OTT T, ROBINSON G, and HENNIGHAUSEN L (2004). Loss of connexin 26 in mammary epithelium during early but not during late pregnancy results in unscheduled apoptosis and impaired development. Dev Biol 267: 418-429.

CARYSTINOS G, BIER A, and BATIST G (2001). The role of connexin-mediated cell-cell communication in breast cancer metastasis. J Mammary Gland Biol Neoplasia 6: 431-440.

CZYZ J (2008). The stage-specific function of gap junctions during tumourigenesis. Cell Mol Biol Lett 13: 92-102.

DBOUK H, MROUE R, EL-SABBAN M, and TALHOUK R (2009). Connexins: A myriad of functions extending beyond assembly of gap junction channels. Cell Commun Signal 7: 4.

EL-SABBAN M, ABI-MOSLEH L, and TALHOUK R (2003a). Developmental regulation of gap junctions and their role in mammary epithelial cell differentiation. $J$ Mammary Gland Biol Neoplasia 8: 463-473.

EL-SABBAN M, SFEIR A, DAHER M, KALAANY N, BASSAM R, and TALHOUK $R$ (2003b). ECM-induced gap junctional communication enhances mammary epithelial cell differentiation. J Cell Sci 116: 3531-3541.

ELZARRAD M, HAROON A, WILLECKE K, DOBROWOLSKI R, GILLESPIE M, and AL-MEHDI A (2008). Connexin-43 upregulation in micrometastases and tumor vasculature and its role in tumor cell attachment to pulmonary endothelium. BMC Med 6: 20.

GAKHAR G, HUA D, and NGUYEN T (2010). Combinational treatment of gap junctional activator and tamoxifen in breast cancer cells. Anticancer Drugs 21: 77-88.

HEINIGER B, GAKHAR G, PRASAIN K, HUA D, and NGUYEN T (2010). Secondgeneration substituted quinolines as anticancer drugs for breast cancer. Anticancer Res 30: 3927-3932.

JIANG W, BRYCE R, and MANSEL R (1997). Gamma linolenic acid regulates gap junction communication in endothelial cells and their interaction with tumour cells. Prostaglandins Leukot Essent Fatty Acids 56: 307-316.

KALRAJ, SHAOQ, QINH, THOMAST, ALAOUI-JAMALIM, and LAIRD D (2006). Cx26 inhibits breast MDA-MB-435 cell tumorigenic properties by a gap junctional intercellular communication-independent mechanism. Carcinogenesis 27:2528-2537.

KANCZUGA-KODAL, SULKOWSKAM, KODAM, RUTKOWSKI R, and SULKOWSKI $S$ (2007). Increased expression of gap junction protein--connexin 32 in lymph node metastases of human ductal breast cancer. Folia Histochem Cytobiol 45 Suppl 1: S175-80.

KANCZUGA-KODA L, SULKOWSKI S, LENCZEWSKI A, KODA M, WINCEWICZ A, BALTAZIAK M, and SULKOWSKA M (2006). Increased expression of connexins 26 and 43 in lymph node metastases of breast cancer. J Clin Pathol 59: 429-433.

KAPOOR P, SAUNDERS M, LI Z, ZHOU Z, SHEAFFER N, KUNZE E, SAMANT R, WELCH D, and DONAHUE H (2004). Breast cancer metastatic potential: Correlation with increased heterotypic gap junctional intercellular communication between breast cancer cells and osteoblastic cells. Int J Cancer 111: 693-697.
KHOKHAR and WERBZ (2011). Mammary gland reprogramming: Metalloproteinases couple form with function. Cold Spring Harb Perspect Biol 3: a004333.

LAIRD D (2010). The gap junction proteome and its relationship to disease. Trends Cell Biol 20: 92-101.

LANIGAN F, O'CONNOR D, MARTIN F, and GALLAGHER W (2007). Molecular links between mammary gland development and breast cancer. Cell Mol Life Sci 64: 3159-3184.

LELIEVRE S (2010). Tissue polarity-dependent control of mammary epithelial homeostasis and cancer development: An epigenetic perspective. J Mammary Gland Biol Neoplasia 15: 49-63.

LI Z, ZHOU Z, WELCHD, and DONAHUE H (2008). Expressing connexin 43 in breast cancer cells reduces their metastasis to lungs. Clin Exp Metastasis 25: 893-901.

LOCKE D (1998). Gap junctions in normal and neoplastic mammary gland. J Pathol 186: 343-349.

LOCKE D, JAMIESON S, STEIN T, LIU J, HODGINS M, HARRIS A, and GUSTERSON B (2007). Nature of Cx30-containing channels in the adult mouse mammary gland. Cell Tissue Res 328: 97-107.

LOCKED, PERUSINGHEN, NEWMANT, JAYATILAKE H, EVANSW, and MONAGHAN $P(2000)$. Developmental expression and assembly of connexins into homomeric and heteromeric gap junction hemichannels in the mouse mammary gland. J Cell Physiol 183: 228-237.

LOCKE D, STEIN T, DAVIES C, MORRIS J, HARRIS A, EVANS W, MONAGHAN P, and GUSTERSON B (2004). Altered permeability and modulatory character of connexin channels during mammary gland development. Exp Cell Res 298: 643-660.

LOEWENSTEIN W and KANNO Y (1966). Intercellular communication and the control of tissue growth: Lack of communication between cancer cells. Nature 209: 1248-1249.

MALLER O, MARTINSON H, and SCHEDIN P (2010). Extracellular matrix composition reveals complex and dynamic stromal-epithelial interactions in the mammary gland. J Mammary Gland Biol Neoplasia 15: 301-318.

MCLACHLAN E, SHAO Q, and LAIRD D (2007). Connexins and gap junctions in mammary gland development and breast cancer progression. $J$ Membr Biol 218: 107-121.

MCLACHLAN E, SHAO Q, WANG H, LANGLOIS S, and LAIRD D (2006). Connexins act as tumor suppressors in three-dimensional mammary cell organoids by regulating differentiation and angiogenesis. Cancer Res 66: 9886-9894.

MESNILM and YAMASAKIH (2000). Bystander effect in herpes simplex virus-thymidine kinase/ganciclovir cancer gene therapy: Role of gap-junctional intercellular communication. Cancer Res 60: 3989-3999.

MOMIYAMA M, OMORI Y, ISHIZAKI Y, NISHIKAWA Y, TOKAIRIN T, OGAWA J, and ENOMOTO K (2003). Connexin26-mediated gap junctional communication reverses the malignant phenotype of MCF-7 breast cancer cells. Cancer Sci 94: 501-507.

MROUE R, EL-SABBAN M, and TALHOUK R (2011). Connexins and the gap in context. Integr Biol (Camb) 3: 255-266.

NAOI Y, MIYOSHI Y, TAGUCHIT, KIM S, ARAI T, TAMAKIY, and NOGUCHI S (2007). Connexin26 expression is associated with lymphatic vessel invasion and poor prognosis in human breast cancer. Breast Cancer Res Treat 106: 11-17.

NAUS C and LAIRD D (2010). Implications and challenges of connexin connections to cancer. Nat Rev Cancer 10: 435-441.

PLANTE I and LAIRD D (2008). Decreased levels of connexin43 result in impaired development of the mammary gland in a mouse model of oculodentodigital dysplasia. Dev Biol 318: 312-322.

PLANTE I, STEWART M, BARR K, ALLAN A, and LAIRD D (2011). Cx43 suppresses mammary tumor metastasis to the lung in a $\mathrm{C} \times 43$ mutant mouse model of human disease. Oncogene. 30: 1681-1692.

PLANTE I, WALLIS A, SHAO Q, and LAIRD D (2010). Milk secretion and ejection are impaired in the mammary gland of mice harboring a $\mathrm{Cx} 43$ mutant while expression and localization of tight and adherens junction proteins remain unchanged. Biol Reprod 82: 837-847.

POLLMANN M, SHAO Q, LAIRD D, and SANDIG M (2005). Connexin 43 mediated gap junctional communication enhances breast tumor cell diapedesis in culture. Breast Cancer Res 7: R522-R534.

QINH, SHAO Q, CURTISH, GALIPEAUJ, BELLIVEAUD, WANG T, ALAOUI-JAMALI $M$, and LAIRD D (2002). Retroviral delivery of connexin genes to human breast 
tumor cells inhibits in vivo tumor growth by a mechanism that is independent of significant gap junctional intercellular communication. JBiol Chem277:29132-29138.

QIN H, SHAO Q, THOMAS T, KALRA J, ALAOUI-JAMALI M, and LAIRD D (2003). Connexin26 regulates the expression of angiogenesis-related genes in human breast tumor cells by both GJIC-dependent and -independent mechanisms. Cell Commun Adhes 10: 387-393.

SAEZ C, VELASQUEZ L, MONTOYA M, EUGENIN E, and ALVAREZ M (2003). Increased gap junctional intercellular communication is directly related to the anti-tumor effect of all-trans-retinoic acid plus tamoxifen in a human mammary cancer cell line. J Cell Biochem 89: 450-461.

SAUNDERS M, SERAJ M, LI Z, ZHOU Z, WINTER C, WELCH D, and DONAHUE $H$ (2001). Breast cancer metastatic potential correlates with a breakdown in homospecific and heterospecific gap junctional intercellular communication. Cancer Res 61: 1765-1767.

SCHEDIN P and KEELY P (2011). Mammary gland ECM remodeling, stiffness, and mechanosignaling in normal development and tumor progression. Cold Spring Harb Perspect Biol 3: a003228.
SHAOQ, WANGH, MCLACHLANE, VEITCHG, and LAIRDD (2005). Down-regulation of $\mathrm{Cx43}$ by retroviral delivery of small interfering RNA promotes an aggressive breast cancer cell phenotype. Cancer Res 65: 2705-2711.

SOHL G and WILLECKE K (2004). Gap junctions and the connexin protein family. Cardiovasc Res 62: 228-232.

TALHOUK R, ELBLE R, BASSAM R, DAHER M, SFEIR A, MOSLEH L, EL-KHOURY $\mathrm{H}$, HAMOUI S, PAULI B, and EL-SABBAN M (2005). Developmental expression patterns and regulation of connexins in the mouse mammary gland: Expression of connexin30 in lactogenesis. Cell Tissue Res 319: 49-59.

TALHOUK R, MROUE R, MOKALLED M, ABI-MOSLEH L, NEHME R, ISMAIL A, KHALIL A, ZAATARI M, and EL-SABBAN M (2008). Heterocellular interaction enhances recruitment of alpha and beta-catenins and ZO-2 into functional gap-junction complexes and induces gap junction-dependant differentiation of mammary epithelial cells. Exp Cell Res 314: 3275-3291.

TIEDE B and KANG Y (2011). From milk to malignancy: The role of mammary stem cells in development, pregnancy and breast cancer. Cell Res 21: 245-257. 


\section{Further Related Reading, published previously in the Int. J. Dev. Biol.}

\section{Modeling and quantification of cancer cell invasion through collagen type I matrices}

Olivier De Wever, An Hendrix, Astrid De Boeck, Wendy Westbroek,Geert Braems, Shahin Emami, Michèle Sabbah, Christian Gespach and Marc Bracke.

Int. J. Dev. Biol. (2010) 54: 887-896

Epithelial-Mesenchymal Transitions in development and disease: old views and new perspectives M. Angela Nieto.

Int. J. Dev. Biol. (2009) 53: 1541-1547

Cadherin-mediated cell-cell adhesion and tissue segregation in relation to malignancy Ramsey A. Foty and Malcolm S. Steinberg

Int. J. Dev. Biol. (2004) 48: 397-409

$\mathrm{N}$-cadherin in the spotlight of cell-cell adhesion, differentiation, embryogenesis, invasion and signalling Lara D.M. Derycke and Marc E. Bracke

Int. J. Dev. Biol. (2004) 48: 463-476

Analysis of tenascin mRNA expression in the murine mammary gland from embryogenesis to carcinogenesis: an in situ hybridization study.

I Kalembey, T Yoshida, K Iriyama and T Sakakura

Int. J. Dev. Biol. (1997) 41: 569-573

$$
5 \text { yr ISI Impact Factor }(2010)=2.961
$$

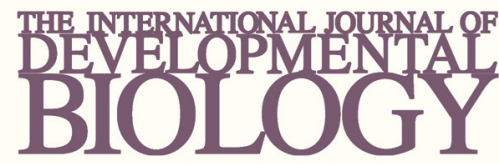

Volume 54 Nos. 6/7
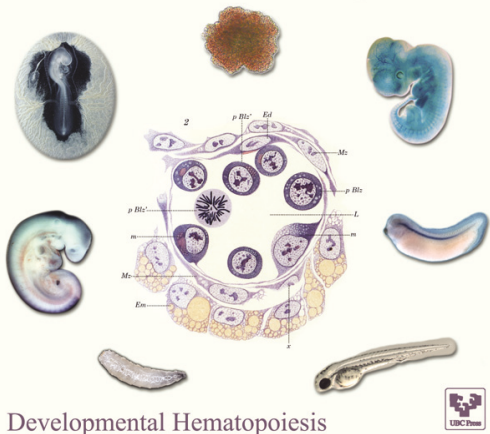

Developmental Hematopoiesis
Special Issue
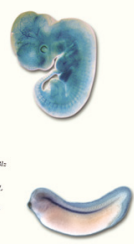

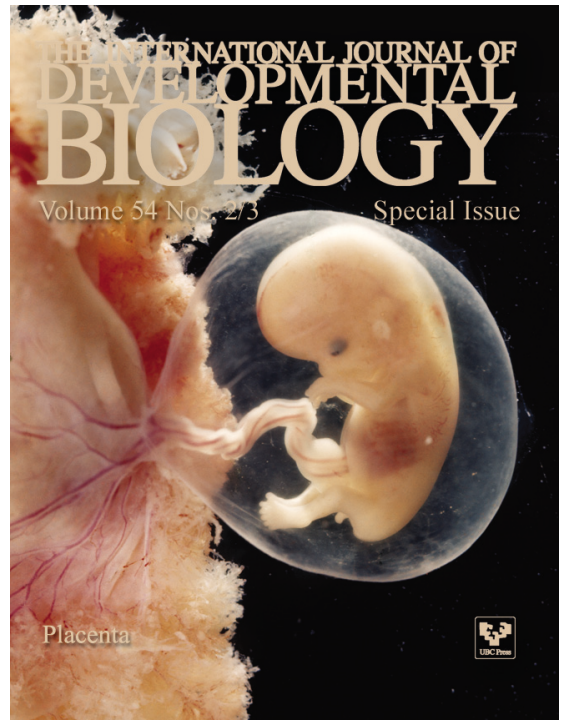

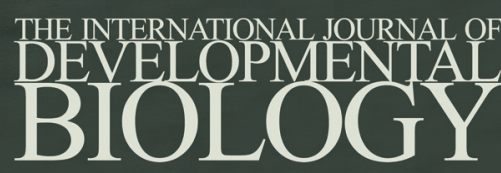

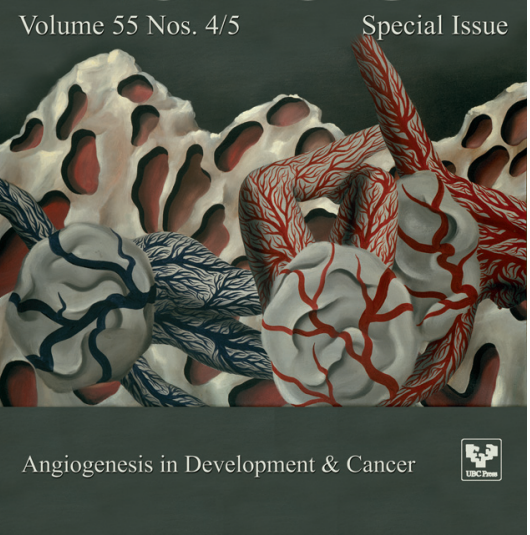

